Action" document. An Aboriginal community member also wrote a culturally aligned, plain language version.

Results In 6 months, peer-supported recommendations were developed and broadly disseminated to stakeholders locally and nationally. The "Call To Action" was distributed to stakeholders via facilitated discussions, presentations, email, and internet. A followup survey of stakeholders will be conducted to assess the impact of our dissemination approach. The intended outcomes will include increased awareness, knowledge, and investment in evidenceinformed strategies as recommended in the "Call To Action".

Conclusions The approach undertaken provided timely research evidence for policy makers. Other than raising awareness, the impact of this approach remains to be determined.

\section{SP3-75 TRAINING IN EPIDEMIOLOGY IN EUROPE}

doi:10.1136/jech.2011.1429760.75

${ }^{1} \mathrm{C}$ Junker, ${ }^{* 2}{ }^{2}$ Luostarinen. ${ }^{1}$ Federal Statistical Office, Neuchâtel, Switzerland; ${ }^{2}$ Finnish Cancer Registry, Helsinki, Finland

Training in Epidemiology in Europe is evolving. The European Epidemiology Federation IEA-EEF felt the need to know more about existing training possibilities and therefore conducted a survey "Degrees in Epidemiology" with the aim to create an inventory of academic institutions offering formal training in Epidemiology.

Methods Online Survey starting in April 2010, still going on.

Results Up to December 2010 eleven universities gave information on programs leading to an MSc and/or $\mathrm{PhD}$ in Epidemiology. Several programs started between 2006 and 2008. We estimate, that there are more than 300 students enrolled each year in European programs. An update of this survey will be presented at the conference.

Conclusion When for many years the possibilities to study Epidemiology in Europe were restricted to very few well known places and many students went overseas, there are now increasing possibilities to study epidemiology "at home". This may help to strengthen our discipline.

\section{SP3-76 INCIDENCE AND PERSISTENCE OF OBESITY IN BRAZILIAN ADULTS FROM BODY MASS INDEX AT END OF ADOLESCENCE}

doi:10.1136/jech.2011.1429760.76

W Conde, ${ }^{*}$ C Borges. Public Health of School, University of Sao Paulo, Sao Paulo, Sao Paulo, Brazil

Introduction The prevalence of obesity has increased among adults living in developed or developing countries. In Brazil, obesity among adults reached at least $10 \%$ of population in 2008-2009. This study estimates the incidence and persistence of overweight or obesity in adults according to nutritional status at end of adolescence.

Methods Former and present self-reported anthropometric data come from VIGITEL 2006 to 2009 (acronym in Portuguese for System of Health Surveillance by Phone) and include 61985 cases from individuals aged 21 to 39 years. Nutritional status was classified according to WHO systems. The RR for obesity was calculated according to level of education.

Results For individual with normal BMI at 20 years incidence of overweight is $40 \%$ among male and $30 \%$ among female. For individuals with pre-obese BMI at 20 years the incidence of obesity is 5.4 higher among male and 4.7 higher among females than that for normal BMI at 20 years. Persistence of overweight is estimated in $91 \%$ of males and $81 \%$ of females. For individuals with pre-obese BMI at 20 years the incidence of obesity is 5.4 higher for males and 4.7 higher for females than that for normal BMI at 20 years.
Persistence of obesity from 20 years to present is about $60 \%$ among males and $49 \%$ among females. RR of present obesity among college degree vs $<4$ years of school education, both with normal BMI at 20 years, is 0.56 for males and 0.26 for females.

Conclusion Higher level of education is associated with a lower incidence and persistence of obesity among adults, especially among females.

\section{SP3-77 PROCESSED MEAT CONSUMED BY BRAZILIAN ADOLESCENTS: AN ANALYSIS ACCORDING TO TRAFFIC LIGHT LABELLING}

doi:10.1136/jech.2011.1429760.77

A M de Carvalho, E V Junior, A Previdelli, B Gorgulho, D Marchioni, ${ }^{*}$ R Fisberg. University of São Paulo, São Paulo, Brazil

Introduction The consumption of processed foods is associated with chronic diseases and obesity. Despite nutritional information on the label being mandatory in Brazil, this information is poorly understood by the population. The use of Traffic Light Labelling (TLL) may be a good alternative to help consumers with their choices. This study analysed processed meats consumed by Brazilian adolescents according to the UK regulation (TLL).

Methods In 2008, a population-based survey was conducted in São Paulo, Brazil. Dietary data from 170 adolescents were obtained through one 24-h dietary recall. The TLL uses traffic light signals: red (high), yellow (medium) and green (low), to represent the percentages of sugar, total and saturated fat and salt in pre-packed foods. The content of salt, total and saturated fat was calculated for processed meats consumed.

Results The processed meats represented $27 \%$ of total meat consumed. Almost $80 \%$ of processed meats had more sodium and $50 \%$ more saturated and total fat than the maximum recommended by TLL regulations, for example, bacon has more than four times the amount of sodium and twice the amount of total and saturated fat than TLL. The most frequently consumed processed meats were pork sausage, hamburger, nuggets, mortadella and sausage. Among these foods, pork sausage, nuggets, mortadella and sausage should be presented with a red colour on the packet for the all nutrients and the hamburger should be yellow, indicating which of these foods should be avoided. Conclusion These results emphasise the need for specific regulation for processed foods, especially processed meats in order to avoid the burden of chronic diseases.

\section{SP3-78 VALIDITY AND REPRODUCIBILITY OF A FOOD FREQUENCY QUESTIONNAIRE DEVELOPED BASED ON A REPRESENTATIVE SAMPLE OF ADOLESCENTS FROM SÃO PAULO, BRAZIL}

doi:10.1136/jech.2011.1429760.78

D Marchioni, ${ }^{*}$ A Carvalho, J Carlos, S Selem, J Teixeira, E Verly Jr, R Fisberg. São Paulo University, São Paulo, São Paulo, Brazil

Introduction The food frequency questionnaire (FFQ) has been one the most widely used method for assessment of food consumption, as well as being able to provide the usual intake, is considered easy to use and of low cost.

Objective To assess the validity and reproducibility of a quantitative FFQ with 66 food items (OFFQ) developed based on a representative sample of adolescents from São Paulo, Brazil.

Methods 250 adolescents filled two FFQ (1-year interval) and 5 24-h recalls (3-month interval) as part of a population-based survey conducted in São Paulo, Brazil, in 2007. To quantify the nutrient intake, the software Nutrition Data System for Research was used. 
The intake was adjusted for within-person variability by the method proposed by lowa State University, using the software PCSIDE, and energy-adjusted for the residual method. The validity for 23 nutrients was assessed by Spearman correlation coefficient and weighted $\kappa$. Intra-class correlation coefficient and weighted $\kappa$ was used to reproducibility assessment.

Results In the validation, the Spearman correlation coefficients ranged from 0.25 (riboflavin) to 0.57 (phosphorus) of which eight nutrients showed acceptable correlation $(>0.4)$. In the reproducibility analysis, the intra-class correlation coefficients ranged from 0.18 (vitamin C) to 0.57 (niacin), of which five nutrients showed acceptable correlation. The weighted $\kappa$ ranged from 0.18 (sodium) to 0.67 (riboflavin) to reproducibility and from 0.19 (polyunsatured fat) to 0.56 (calcium) to validation.

Conclusion The results support the use of this instrument to assess food intake in epidemiological studies conducted in São Paulo, Brazil.

\section{SP3-79 AUDIT OF A NATIONWIDE PATHOLOGY-BASED CANCER REGISTRY IN IRAN AND LESSONS LEARNED FROM SUCCESSFUL POPULATION-BASED CANCER REGISTRIES WORLDWIDE}

doi:10.1136/jech.2011.1429760.79

K Zendehdel, G Keshtmand, * Z Sedighi, J Hassanloo, A Nahvijou, M A Mohagheghi. Cancer Research Center, Cancer Institute of Iran, Tehran University of Medical Sciences, Tehran, Iran

Background Several low- and middle- income countries, lack wellfunctioning population-based cancer registry. We evaluated the completeness of a pathology based cancer registry in Iran. We further studies evolutionary progress of the cancer registries worldwide.

Methods We evaluated consistency of the incidence rates reported by national pathology-based cancer registry in Iran from 2004 to 2007. We further compared the incidence rates of the pathology- and population-based registries in a few regions, where both data were available. In addition, we studied the increasing trend in the number of population-based cancer registries worldwide, using the reports published in the Volumes I-IX of the monograph "Cancer in Five Continents."

Results The Iranian pathology-based cancer registry, reports only about $60-70 \%$ of cancers. The underestimates were greater in cancers with poor-prognosis including lung, stomach, and oesophageal cancers. Almost four regional cancer registries were established every 10 years since 1960. However, the USA was an exception, where the number of cancer registries increased from 14 in 1998 to 44 regional registries in 2002, due to the advance infrastructure in the health informatics and ambitious initiatives by the Centers for Disease Control in the USA.

Conclusions Pathology based cancer registry cannot provide reliable estimate for the cancer incidence rates, particularly in cancers with a poor prognosis. Developing countries should establish and support regional registries and expand their coverage gradually. Given the recent advances in the health informatics, small efforts will enhance the coverage of cancer registries worldwide, particularly in the less than middle income countries.

\section{SP3-80 PARENT'S SOCIAL STATUS AND EARLY NUTRITION INFLUENCES ON COLLEGE ENTRANCE AMIONG BRAZILIAN YOUTH AT 2 DECADES}

doi:10.1136/jech.2011.1429760.80

L Baraldi, ${ }^{*}$ W Conde. Public Health School, São Paulo, São Paulo, Brazil

Introduction Human capital concept is related to social and nutrition experiences in childhood as well as social status inherited from their parents.
Objective Estimate the chance of university achievement among Brazilian youth with adequate or inadequate early nutrition and born from parents with low vs high school degree.

Methods A sample of young, aged 20-24 years, and their parents from three Brazilian surveys, PNSN-1989, POF-2003 and POF-2009. Nutritional status was standardised from WHO reference2007; highest school level achieved was used to classify social status. We set three nutrition groups (below $-1 Z,-1$ to +1 , and above $+1 Z$, named N1, N2 and N3, respectively) for parents and youth and three school levels groups (elementary, high, college, named E1, E2, E3 respectively) for parents. Probability of beginning university by youth was estimated using multiple logistic regression. Survey year was included as independent dummy variable to estimate changes among periods.

Results The ratio between parents E3 vs E1 among youth N1 was 7.0 in 1989, 11.0 in 2003 and 6.1 in 2009. This ratio for young N2 was 5.9 in 1989, 9.7 and 5.0 in 2003 and 2009, respectively. For young's N3 that ratio was 3.9 in 1989, 8.8 in 2003 and 4.0 in 2009. Conclusion The unequal chance of being university student in Brazil as function of parent's social status and early nutrition decreased from period 1989-2003 to period 2003-2009. Probably this change is associated with social mobility experienced in country this decade.

\section{SP3-81 GEO-EPIDEMIOLOGY OF MULTIDRUG-RESISTANT TUBERCULOSIS CASES IN THE STATE OF SÃO PAULO, BRAZIL, 2007-2010}

doi:10.1136/jech.2011.1429760.81

P A Opromolla, ${ }^{*}$ V M Galesi, L A R dos Santos, M J P Rujula. Secretaria de Estado da Saúde, São Paulo, São Paulo, Brazil

In São Paulo, resistant tuberculosis (MDR-TB) is defined for the purpose of notification and treatment, as those cases that present with in-vitro resistance to rifampicin and isoniazid, and a third standard drug. There is currently a system of epidemiological surveillance of MDR-TB, which monitors all re-treatment cases of the disease nationwide. In the State of São Paulo, this monitoring is done by the division of tuberculosis Epidemiological Surveillance Centre of São Paulo. The aim of this study was to analyse the spatial distribution of cases of resistant tuberculosis in the state of São Paulo, Brazil, in the period 2007-2010.

Methods We selected confirmed cases of MDR-TB, notified to the state of Sao Paulo in the period 1 January 2007 to 10 January 2011 with the county as the unit of spatial analysis. We also analysed data from incident cases of tuberculosis in the state during the same period and with the same spatial unit of analysis. In addition to these data, information about detention facilities was also collected. All data were analysed in GIS, using space techniques for the detection of clusters and spatial correlations.

Results We detected 355 cases of MDR-TB during the study period, $34 \%$ in $2010,68.7 \%$ male, $63 \%$ Caucasian and $77 \%$ by 11 years of study. The mean age was 38 years ( \pm 12 years). $97 \%$ were of pulmonary disease, only $11 \%$ were HIV-positive. Using kernel parameters, the greatest probability of MDR-TB cases are concentrated in the southeastern state.

\section{SP3-82 FACTORS ASSOCIATED WITH ORAL HEALTH STATUS OF ADULTS, BRAZIL}

doi:10.1136/jech.2011.1429760.82

${ }^{1} \mathrm{~F}$ Floriano, ${ }^{*} \mathrm{C} C$ Costa Mda, ${ }^{2} \mathrm{M}$ I Vianna, ${ }^{2} \mathrm{M}$ B Cabral, ${ }^{2} \mathrm{M}$ dos Santos, ${ }^{1} \mathrm{G}$ Santos. ${ }^{1}$ ISC-UFBA, SSA, Bahia, Brazil; ${ }^{2}$ FO-UFBA, SSA, Bahia, Brazil

Introduction The objective of this study was to evaluate the oral health status of adults in Salvador-Bahia, Brazil, and to identify 